\title{
Diseño y construcción de un instrumento para evaluar el potencial de habilidades directivas de los trabajadores
}

\section{Design and construction of an instrument to evaluate the potential of employees management skills}

\begin{abstract}
RESUMEN
El artículo tuvo como propósito el diseño y la construcción de un instrumento (test) para evaluar el potencial de las habilidades directivas de los trabajadores, considerando las dimensiones: desarrollo del autoconocimiento, manejo del estrés y del bienestar, solución creativa de problemas, manejo de conflictos, motivación a los trabajadores, comunicación de apoyo, ganar poder e influencia, formación de equipos efectivos, liderar el cambio positivo y facultamiento y delegación. La investigación es de tipo instrumental. enfoque cuantitativo, diseño no experimental de tipo transversal y de nivel descriptivo. Los 50 ítems del instrumento en construcción se sometieron a Juicio de Expertos y se obtuvo el valor promedio del Coeficiente V de Aiken $=0.962$, que indica alta validez de contenido. En la correlación Ítem - Correlación total de elementos corregidos, se obtuvo valores entre 0.40 y 0.69 , significando que el test tiene validez interna. La confiabilidad se evaluó mediante el método de coeficiente de consistencia interna, aplicando el test a una muestra de 1874 trabajadores de 61 empresas de Lima Metropolitana, cuyo Coeficiente Alfa de Cronbach fue 0.967, que denota una muy fuerte confiabilidad. Asimismo, se elaboró el baremo mediante el percentil de las dimensiones. En conclusión, se cumplió satisfactoriamente la construcción del Test Potencial de Habilidades Directivas, altamente válido y confiable.
\end{abstract}

Palabras claves: Habilidades directivas; dimensiones; validez; confiabilidad; baremo.

\begin{abstract}
The purpose of this article was the design and construction of an instrument (test) to evaluate the potential of workers' managerial skills, considering the dimensions: development of self-knowledge, stress management and well-being, creative problem solving, management of conflict, worker motivation, supportive communication, gaining power and influence,
\end{abstract}

Walter Hugo Arana Mayorca

walter.arana@unmsm.edu.pe ORCID: https://orcid.org/00000003-0700-4322

Universidad Nacional Mayor de San Marcos. Lima, Perú

Presentado: 03/03/2021 - Aceptado: 19/03/2021 - Publicado: 17/06/2021

(C) Los autores. Este artículo es publicado por la revista Gestión en el Tercer Milenio de la Facultad de Ciencias Administrativas Universidad Nacional Mayor de San Marcos. Este es un artículo de acceso abierto, distribuido bajo los términos de la licencia Creative Commons Atribución 4.0 Internacional (CC BY 4.0) [https://creativecommons.org/licenses/by/4.0/deed.es] que permite el uso, distribución y reproducción en cualquier medio, siempre que la obra original sea debidamente citada de su fuente original. 
building effective teams, leading positive change, and empowerment and delegation. The research is instrumental, with a quantitative approach, a non-experimental cross-sectional design and a descriptive level. The 50 items of the instrument under construction were subjected to Expert Judgment and the average value of the Aiken Coefficient $V=0.962$ was obtained, which indicates high content validity. In the Item correlation - Total correlation of corrected items, values between 0.40 and 0.69 were obtained, meaning that the test has internal validity. Reliability was evaluated using the internal consistency coefficient method, applying the test to a sample of 1874 workers from 61 companies in Metropolitan Lima, whose Cronbach's Alpha Coefficient was 0.967 , which denotes a very strong reliability. Likewise, the scale was prepared using the percentile of the dimensions. In conclusion, the construction of the Test Potential Management Skills was satisfactorily completed because the results were highly valid and reliable.

Keywords: Management skills; dimensions; validity; reliability; scale.

\section{INTRODUCCIÓN}

En los últimos tiempos, en parte, por la nueva cultura laboral de los millennials y la generación $\mathrm{Z}$, quienes no aspiran permanencia estable en el trabajo, las empresas están afrontando una alta rotación de personal y están obligadas a realizar estrategias de retención de personal o frecuentes procesos de selección de personal.

Una estrategia importante para retener al personal, va más allá del clima organizacional y las remuneraciones, es Ia oportunidad de crecimiento. En un estudio realizado por Arana (2018) donde participaron 1,214 trabajadores, la mayoría de los encuestados (18,23\%), de las 10 dimensiones evaluadas, consideran que el crecimiento es el factor que más les motiva dentro de su empresa. Por lo tanto, generar condiciones para el crecimiento profesional y personal de los trabajadores en la organización es una acertada decisión, donde la empresa se asegura contar con el talento humano y el trabajador tiene una oportunidad de desarrollo. Identificar el potencial de talentos naturales, habilidades y destrezas de las personas en las organizaciones, es una buena medida (Maxwell, 2018).

Pereda (2016) realizó un estudio sobre las habilidades directivas aplicando al sector público de la provincia de Córdoba (España) haciendo uso de un cuestionario, adaptado de González Santa Cruz, que consta de 27 preguntas e identifica 22 habilidades directivas. Por otro lado, Dincer y Akgum (2015) desarrollaron una herramienta de medición para determinar las habilidades directivas en el ámbito educativo de Turquía, donde participaron 520 docentes en el inventario. Abadi (2013) construyó una escala sobre los componentes de las habilidades de gestión integral de la calidad, donde participaron 250 gerentes mediante un muestreo aleatorio.

En el Perú se realizaron investigaciones sobre habilidades directivas para evaluar a jefes y gerentes, todos ellos con muestras menores de 100 encuestados; por lo tanto, el instrumento utilizado es válido para el grupo materia de evaluación, más no puede ser utilizado en otros grupos de la población. Ninguno de ellos evalúa el potencial.

\section{Dimensiones del Potencial de Habilida- des Directiva}

Whetten y Cameron (2016) consideran que la habilidad directiva es definida como la capacidad que tiene la persona para realizar actividades de gestión y dirección producto de la aptitud incrementada por la capacitación y la oportunidad, basado en dimensiones: personal, interpersonal y grupal.

Teniendo como referencia el Assesment Management Skill - PAMS de Whetten y Cameron (2016), a continuación, se desarrolla las principales dimensiones:

\section{Desarrollo del Autoconocimiento}

Brouwer (cit. Whetten y Cameron, 2016) señala que la autoevaluación es base de la introspección, del análisis de sí mismo, de la toma de conciencia. La introspección es el sentimiento 
de "ah, ahora lo veo", expresión de una visión genuina y real de uno mismo, de cómo somos en realidad, que se logra de manera consciente $o$ inconsciente.

Es importante que el futuro directivo tenga como competencia la toma de conciencia de sus fortalezas y debilidades personales, práctica de respeto a las normas laborales, solicitar apoyo para mejorar aspectos personales, capacidad de escuchar a los demás antes de tomar decisiones y predisposición de adaptación a situaciones ambiguas e inciertas.

\section{Manejo del Estrés y del Bienestar}

Selye (1956) señala que el estrés es un conjunto de fuerzas que regulan funciones que afrontan las amenazas al equilibrio del organismo vivo.

Una persona que acceda a cargos directivos dentro de una organización deberá tener competencias o habilidades personales para enfrentar y superar estados de estrés. Esto implica que la persona practique algún método o ejercicio para el control del estrés, manejo del tiempo, equilibrio en su vida, diferencia entre lo más importante y urgente, así como mantiene una relación sincera y de confianza con personas de su familiaridad.

\section{Solución Creativa de Problemas}

Muchos trabajadores, con el transcurrir del tiempo, sus actividades se convierten en rutinarias, que cuando se presenta un problema tienen dificultades en encontrar una solución por estar encuadrado en una sola forma de trabajar. Según Budjac (2011) la creatividad es la habilidad de ver lo que otros no ven.

El aspirante a ocupar un cargo directivo debe tener competencias personales para plantear clara y explícitamente el problema, poseer alternativas de solución, tener en cuenta puntos de vista divergentes y promueve, de ser el caso, romper con las reglas con el fin de obtener soluciones creativas.

\section{Manejo de Conflictos}

En el ámbito laboral, los problemas están presente principalmente en el factor humano, porque es la decisión o el comportamiento del hombre que provoca éxitos o fracasos en la gestión.
Según Johnson y Keddy (2015) los elementos que subyacen al surgimiento de los conflictos en el trabajo por lo general es imprescindible; por lo tanto, es importante una constante negociación, que según Lax y Sebennius (2007) significa dominar técnicas que se basan en comunicaciones efectivas y destrezas interpersonales.

El aspirante a un cargo directivo debe tener competencias cuyo comportamiento se caracterice por demostrar una actitud serena, ayudar a las partes para solucionar un problema, maneja apropiadamente situaciones conflictivas y sabe escuchar, promueve el diálogo y obtiene resultados satisfactorios.

\section{Motivación a los Trabajadores}

Stonner, Freeman y Gilbert Jr. señalan que: "La motivación es una característica de la psicología humana que contribuye al grado de compromiso de Ia persona". En ese mismo contexto, Gordon (1997) indica que todos los administradores enfrentan un reto enorme: motivar a los empleados para que produzcan los resultados deseados, con eficiencia, calidad e innovación.

El potencial directivo debe tener habilidades para: reconocer oportunamente el logro o el desempeño excepcional de los trabajadores; motivar con entusiasmo a sus compañeros para darles sentido de pertenencia en su organización; ayudar a sus compañeros a lograr las metas, así sean desafiantes; retroalimentar inmediatamente al compañero de trabajo de manera oportuna; y se debe asegurar que Ia gente se sienta tratada con justicia y equidad.

\section{Comunicación de Apoyo}

Por su naturaleza las personas están en permanente interacción con otros, siendo Ia comunicación el medio que los vincula. Por lo tanto, en el ámbito laboral, la comunicación es fundamental para dar o recibir instrucciones, realizar consultas, expresar iniciativas, realizar informes. Blanchard (2016) considera que el motivador número uno de las personas es la retroalimentación sobre los resultados.

El futuro directivo debe tener habilidades para: ayudar a reconocer y definir problemas de los demás; apoya constante al compañero que lo necesita; aconseja oportunamente; en Ia retroalimentación que practica se centra en los 
problemas y no en las personas; y su comunicación clara y concisa.

\section{Ganar Poder e Influencia}

El hecho que los trabajadores reconozcan y valoren el liderazgo o competencia especiales de otro trabajador es importante, porque de manera espontánea hay personas que sobresalen y se distinguen de los demás. Esto genera respeto, especial consideración y sobre todo poder para influir en los demás.

La persona potencial para ocupar un cargo directivo debe tener: habilidad de superación continua para mejorar la calidad de sus habilidades y conocimientos; dialogar incluso con personas que demuestra alta presión, saber reconocer al trabajador que logra objetivos o comparte información relevante; en lo posible sabe evitar amenazas o exigencias para imponer su voluntad a los demás; y debe ser partícipe de actividades o eventos que se realizan a nivel de Ia organización.

\section{Formación de Equipos Efectivos}

Un equipo de trabajo es un conjunto de personas quienes aportan sus conocimientos, experiencia, habilidades, opiniones, etc. para crear sinergia a fin de tomar decisiones orientados al logro de objetivos que interesa a todos. Schein (1982) señala que el concepto de organización procede del hecho de que el individuo es incapaz de cumplir todas sus necesidades y deseos por sí mismo, tienen que basarse en los demás para cumplir sus propias necesidades.

Un trabajador con potencial para ocupar un cargo directivo debe tener competencias orientadas a: saber cómo establecer credibilidad e influencia en los miembros del equipo; conocer maneras de cohesionar a los miembros del equipo; tener maneras de conformación y conducción de equipos; facilitar la realización de actividades en el equipo; y predisposición de contribuir con sus conocimientos, habilidades y experiencia a logro de objetivos comunes en el equipo.

\section{Liderar el Cambio Positivo}

El liderazgo comprendido como "el actuar de las personas en condiciones de cambio; es decir, cuando las organizaciones son dinámicas y están experimentando una transformación, las personas exhiben liderazgo" (Whetten y Cameron, 2016). Según Goleman (2017) en todo proceso de cambio, los líderes gestionan el significado para el grupo.

El nuevo directivo debe tener competencias para ser partícipe y promotor del cambio organizacional y hacer que la gente se comprometa con los cambios.

\section{Facultamiento y Delegación}

El facultamiento conlleva producir en las otras personas: un sentido de autoconfianza, autodeterminación, relevancia personal, significado confianza (Whetten y Cameron, 2016).

Un potencial directivo debe tener la habilidad de asegurarse de tener la confianza de que otras personas pueden realizar sus tareas de manera óptima, realizar encargos con supervisión, compartir con otros los conocimientos y enseñar para delegar.

\section{MÉTODOS}

\section{Tipo y Diseño de Investigación}

La investigación es de tipo instrumental porque se crea un nuevo instrumento de medición psicométrica (Argumedo, Nóblega, Barrig y Otiniano, 2016) tiene enfoque cuantitativo, diseño no experimental de tipo transversal y es de nivel descriptivo (Hernández, Fernández y Baptista, 2014).

\section{Población y Muestra}

La población elegida estuvo conformada por 1874 trabajadores perteneciente a 61 empresas de Lima Metropolitana, de los cuales 37.51\% son mujeres y $62.49 \%$ son hombres; y respecto al tipo de empresa, $52.19 \%$ son empresas de servicios y $47.81 \%$ empresas de producción.

El tipo de muestra es no probabilística con muestreo intencionado (Alarcón, 2013), dado que los encuestadores (alumnos de maestría de Ia Universidad Nacional Mayor de San Marcos), debidamente capacitados, seleccionaron a los trabajadores (técnicos y profesionales) de las empresas donde laboran y que potencialmente podrían en el futuro asumir cargos directivos.

\section{Procedimiento para la Construcción}

Para la construcción del Test Potencial de Habilidades Directivas se consideraron las 
fases propuestas por Muniz y Fonseca-Pedrero (2019), que se ha basado en los procedimientos establecidos por la International Test Commision - ITC (Muñiz, Elosua y Hambleton, 2013) que recoge los estándares de validación de tests desarrollados por la American Educacional Research Association (AERA), la American Psychological Association (APA) y el National Council on Measurement in Education (NCME):

1. Análisis del problema, identificación de la importancia de elaborar un instrumento y revisión de literatura especializada;

2. Definición de la variable, considerando el marco teórico pertinente;

3. Especificaciones de las dimensiones e indicadores;

4. Construcción de ítems teniendo como referencia a Personal Assesment Management Skill - PAMS de Whetten y Cameron (2016).

5. Construcción de la versión preliminar del test con 4 grados de valoración: nunca (0 puntos), pocas veces (1 punto), regularmente (2 puntos) y casi siempre (3 puntos); además, se contó con Ia opinión de tres expertos en el tema, quienes sugirieron mejoras.

6. Estudio piloto, donde la versión preliminar del test se administró a 20 trabajadores (técnicos y profesionales) no directivos, quienes además de responder al test hicieron recomendaciones que permitió mejorar Ia redacción de los ítems;

7. Establecimiento del test definitivo;

8. Aplicación del test a la muestra establecida;
9. Determinación de las propiedades psicométricas para conocer la validez, confiabilidad y baremo del test; $y$

10. Versión final del test, que incluye el informe de resultados.

\section{RESULTADOS}

Luego de la aplicación definitiva del Test: Potencial de Habilidades Directivas, se obtuvieron los siguientes resultados:

\section{Validez de Contenido}

Anastasi y Urbina (1998) señalan que la validez se refiere al grado en que un instrumento mide lo que se pretende medir. The SAGE Glossary of the Social and Behavioral Sciences considera que la validez de contenido es el grado en que los ítems de un instrumento reflejan el contenido y dominio de lo que se mide (cit. por Hernández y Mendoza, 2018).

Para determinar el grado de validez de contenido del Test Potencial de Habilidades Directivas, se realizó mediante Juicio de Expertos. Siete expertos con grados académicos de maestrías y/o doctorados relacionados con gestión del talento humano, evaluaron la: claridad, pertinencia y relevancia de los ítems. Luego se aplicó el Coeficiente de Validez V de Aiken (Escurra, 1988; Aiken, 1985) a cada ítem del test y por cada dimensión con un nivel de significación estadística del 0.05. En la Tabla 1 se muestran los resultados.

En la Tabla 1, se muestra que $V$ de Aiken de la dimensión personal es: factor claridad $=0.981$, pertinencia $=0.979$ y Relevancia $=0.971$; dimensión interpersonal (claridad $=0.971$, pertinencia $=0.957$ y relevancia $=0.957 ;$ y la dimensión grupal (claridad $=0.952$, pertinencia $=0.929 \mathrm{y} \mathrm{la}$

Tabla 1

Resultados de V de Aiken de las dimensiones del test

\begin{tabular}{lccc}
\hline \multirow{2}{*}{ Dimensiones } & & Factores & Relevancia \\
\cline { 2 - 4 } & Claridad & Pertinencia & 0.971 \\
\hline Personal & 0.981 & 0.979 & 0.971 \\
Interpersonal & 0.971 & 0.957 & 0.952 \\
Grupal & 0.952 & 0.929 & \\
\hline
\end{tabular}

Nota. Elaboración propia 
relevancia $=0.952$ ). El promedio general de $\mathrm{V}$ de Aiken $=0.962$. Es decir, el Test: Potencial de Habilidades Directivas es altamente válido.

Por otro lado, las apreciaciones cualitativas de los expertos se tomaron en cuenta para mejorar la redacción y el orden, que permitió establecer la versión final del test.

\section{Validez Interna}

La validez interna permite analizar y evaluar a cada uno de los ítems o reactivos para establecer la homogeneidad. Para proceder con la validación interna se hace uso de la correlación Ítem - Correlación total de elementos corregida a fin de decidir qué ítems mantener y cuales eliminar. Según Cohen y Manion (2012) se espera que la correlación debe ser $\geq 0.35$ estadísticamente significativo al $1 \%$.

Para conocer Ia validez interna del Test Potencial de Habilidades Directivas, se hizo uso del SPSS (Statistical Package for the Social Sciences) versión 24. Los resultados se muestran en la Tabla 2.

Según se demuestra en la Tabla 2, al analizar la correlación Ítem - Correlación total de elementos corregida de los 50 ítems se obtuvo valores entre 0.40 y 0.69 ; por lo tanto, los 50 ítems se mantuvieron en el test, dado que en todos los casos el valor es superior a 0.35. En consecuencia, el Test Potencial de Habilidades Directivas tiene validez interna.

Tabla 2

Validación interna de los ítems del Test: Potencial de Habilidades Directivas

\begin{tabular}{|c|c|c|c|c|c|c|c|}
\hline $\begin{array}{l}\mathrm{N}^{\circ} \\
\text { ítems }\end{array}$ & $\begin{array}{l}\text { Media de escala si } \\
\text { el elemento se ha } \\
\text { suprimido }\end{array}$ & $\begin{array}{l}\text { Varianza de escala } \\
\text { si el elemento se ha } \\
\text { suprimido }\end{array}$ & $\begin{array}{l}\text { Alfa de Cronbach } \\
\text { si el elemento se } \\
\text { ha suprimido }\end{array}$ & $\begin{array}{l}\mathrm{N}^{\circ} \\
\text { ítems }\end{array}$ & $\begin{array}{l}\text { Media de escala si } \\
\text { el elemento se ha } \\
\text { suprimido }\end{array}$ & $\begin{array}{l}\text { Varianza de escala } \\
\text { si el elemento se ha } \\
\text { suprimido }\end{array}$ & $\begin{array}{l}\text { Alfa de Cronbach } \\
\text { si el elemento se } \\
\text { ha suprimido }\end{array}$ \\
\hline 1 & 96.89 & 576.37 & 0.967 & 26 & 96.5 & 571.88 & 0.966 \\
\hline 2 & 96.88 & 573.82 & 0.966 & 27 & 96.76 & 570.23 & 0.966 \\
\hline 3 & 96.63 & 577.61 & 0.966 & 28 & 96.56 & 570.74 & 0.966 \\
\hline 4 & 96.61 & 571.84 & 0.966 & 29 & 96.79 & 571.37 & 0.966 \\
\hline 5 & 96.56 & 570.75 & 0.966 & 30 & 96.62 & 570.64 & 0.966 \\
\hline 6 & 96.54 & 572.19 & 0.966 & 31 & 96.64 & 569.58 & 0.966 \\
\hline 7 & 96.39 & 574.15 & 0.966 & 32 & 96.48 & 570.95 & 0.966 \\
\hline 8 & 96.62 & 569.72 & 0.966 & 33 & 96.48 & 575.19 & 0.966 \\
\hline 9 & 96.56 & 569.79 & 0.966 & 34 & 96.56 & 572.57 & 0.966 \\
\hline 10 & 96.52 & 572.71 & 0.966 & 35 & 96.7 & 572.72 & 0.966 \\
\hline 11 & 96.7 & 570.73 & 0.966 & 36 & 96.73 & 570.99 & 0.966 \\
\hline 12 & 96.42 & 576.31 & 0.966 & 37 & 96.6 & 572.53 & 0.966 \\
\hline 13 & 96.62 & 571.43 & 0.966 & 38 & 96.6 & 570.62 & 0.966 \\
\hline 14 & 96.6 & 570.26 & 0.966 & 39 & 96.45 & 584.31 & 0.967 \\
\hline 15 & 96.49 & 575.03 & 0.966 & 40 & 96.49 & 573.69 & 0.966 \\
\hline 16 & 96.52 & 572.44 & 0.966 & 41 & 96.66 & 570.3 & 0.966 \\
\hline 17 & 96.46 & 572.34 & 0.966 & 42 & 96.56 & 570.86 & 0.966 \\
\hline 18 & 96.88 & 577.88 & 0.967 & 43 & 96.57 & 572.14 & 0.966 \\
\hline 19 & 96.64 & 570.78 & 0.966 & 44 & 96.51 & 573.69 & 0.966 \\
\hline 20 & 96.82 & 569.81 & 0.966 & 45 & 96.87 & 569.15 & 0.966 \\
\hline 21 & 96.71 & 568.82 & 0.966 & 46 & 96.58 & 568.64 & 0.966 \\
\hline 22 & 96.51 & 572.6 & 0.966 & 47 & 96.38 & 575.61 & 0.966 \\
\hline 23 & 96.78 & 572.33 & 0.966 & 48 & 96.54 & 569.09 & 0.966 \\
\hline 24 & 96.55 & 575.02 & 0.966 & 49 & 96.49 & 574.78 & 0.966 \\
\hline 25 & 96.51 & 574.73 & 0.966 & 50 & 96.52 & 569.5 & 0.966 \\
\hline
\end{tabular}

Nota. Elaboración propia 


\section{Confiabilidad}

Alarcón (2013) considera que el concepto de confiabilidad de un test hace referencia a la consistencia de los puntajes obtenidos por un mismo grupo de sujetos en una serie de mediciones tomadas con el mismo test. La confiabilidad denota estabilidad y constancia de los puntajes.

\section{Coeficiente de Consistencia Interna}

Se utilizó el Coeficiente alfa de Cronbach. Este coeficiente requiere de una sola administración del instrumento de medición y produce valores que oscilan entre 0 y 1 (Hernández, Fernández y Baptista, 1998).

Para determinar el grado de confiabilidad y el baremo se hizo uso del SPSS (Statistical Package for the Social Sciences) versión 24, en el que se procesó los datos y se obtuvo el resultado que se demuestra en la Tabla 3.

El valor obtenido fue Alfa de Cronbach = 0.977; por lo tanto, significa que el Test: Potencial de Habilidades Directivas tiene una muy fuerte confiabilidad.

Los valores del Coeficiente de la correlación total de elementos corregidos correspondientes a los ítems del test presentaron un valor mínimo de 0.40 y un valor máximo de 0.70 (Tabla 4).

\section{Baremación}

Se obtuvo el baremo percentil de las dimensiones del Test: Potencial de Habilidades Directivas (Desarrollo del autoconocimiento, Manejo del estrés y del bienestar, Solución creativa de problemas, Manejo de conflictos, Motivación a los trabajadores, Comunicación de apoyo, Ganar poder e influencia, Formación de equipos efectivos, Liderar el cambio positivo y Facultamiento y delegación). En la Tabla 5 se describe los percentiles (PC), los puntajes directos (PD), la media (M), la desviación estándar (DS) y la varianza (Var).

Tabla 3

Estadística de fiabilidad del Test: Potencial de Habilidades Directivas

\begin{tabular}{cccccc}
\hline Alfa de Cronbach & $\begin{array}{c}\text { Alfa de Cronbach basada en } \\
\text { elementos estandarizados }\end{array}$ & Nro. de elementos & Media & Varianza & Desv. Desviación \\
\hline 0.967 & 0.967 & 50 & 98.57 & 595.605 & 24.405 \\
\hline
\end{tabular}

Nota. Elaboración propia

Tabla 4

Estadístico de correlación total de elementos corregidos

\begin{tabular}{|c|c|c|c|c|c|c|c|}
\hline $\begin{array}{l}\mathrm{N}^{\circ} \\
\text { ítems }\end{array}$ & $\begin{array}{l}\text { Correlación total de } \\
\text { elementos corregidos }\end{array}$ & $\begin{array}{c}\mathrm{N}^{\circ} \\
\text { items }\end{array}$ & $\begin{array}{c}\text { Correlación total de total } \\
\text { de Elementos }\end{array}$ & $\begin{array}{c}\mathrm{N}^{\circ} \\
\text { items }\end{array}$ & $\begin{array}{l}\text { Correlación total de } \\
\text { elementos corregidos }\end{array}$ & $\begin{array}{c}\mathrm{N}^{\circ} \\
\text { items }\end{array}$ & $\begin{array}{l}\text { Correlación total de } \\
\text { elementos corregidos }\end{array}$ \\
\hline 1 & 0.46 & 14 & 0.67 & 27 & 0.63 & 40 & 0.62 \\
\hline 2 & 0.54 & 15 & 0.55 & 28 & 0.66 & 41 & 0.64 \\
\hline 3 & 0.48 & 16 & 0.6 & 29 & 0.54 & 42 & 0.65 \\
\hline 4 & 0.61 & 17 & 0.58 & 30 & 0.66 & 43 & 0.6 \\
\hline 5 & 0.65 & 18 & 0.4 & 31 & 0.68 & 44 & 0.56 \\
\hline 6 & 0.63 & 19 & 0.65 & 32 & 0.64 & 45 & 0.6 \\
\hline 7 & 0.6 & 20 & 0.63 & 33 & 0.59 & 46 & 0.7 \\
\hline 8 & 0.65 & 21 & 0.67 & 34 & 0.63 & 47 & 0.53 \\
\hline 9 & 0.64 & 22 & 0.65 & 35 & 0.6 & 48 & 0.68 \\
\hline 10 & 0.64 & 23 & 0.54 & 36 & 0.65 & 49 & 0.54 \\
\hline 11 & 0.58 & 24 & 0.57 & 37 & 0.64 & 50 & 0.66 \\
\hline 12 & 0.54 & 25 & 0.61 & 38 & 0.66 & & \\
\hline 13 & 0.57 & 26 & 0.63 & 39 & 0.40 & & \\
\hline
\end{tabular}

Nota. Elaboración propia 
Tabla 5

Baremo del Test: Potencial de Habilidades Directivas

\begin{tabular}{|c|c|c|c|c|c|c|c|c|c|c|}
\hline \multirow[b]{2}{*}{ PC } & \multicolumn{10}{|c|}{ PD } \\
\hline & $\begin{array}{l}\text { Desarrollo } \\
\text { del autoco- } \\
\text { nocimiento }\end{array}$ & $\begin{array}{c}\text { Manejo del } \\
\text { estrés y del } \\
\text { bienestar }\end{array}$ & $\begin{array}{l}\text { Solución } \\
\text { creativa de } \\
\text { problemas }\end{array}$ & $\begin{array}{l}\text { Manejo de } \\
\text { conflictos }\end{array}$ & $\begin{array}{c}\text { Motivación } \\
\text { a los traba- } \\
\text { jadores }\end{array}$ & $\begin{array}{c}\text { Comunicación } \\
\text { de apoyo }\end{array}$ & $\begin{array}{c}\text { Ganar } \\
\text { poder e } \\
\text { influencia }\end{array}$ & $\begin{array}{c}\text { Formación } \\
\text { de equipos } \\
\text { efectivos }\end{array}$ & $\begin{array}{l}\text { Liderar } \\
\text { el cambio } \\
\text { positivo }\end{array}$ & $\begin{array}{c}\text { Faculta- } \\
\text { miento y } \\
\text { delegación }\end{array}$ \\
\hline 100 & 15 & 15 & 15 & 15 & 15 & 15 & 15 & 15 & 15 & 15 \\
\hline 80 & 12 & 12 & 12 & 13 & 13 & 13 & 12 & 12 & 13 & 13 \\
\hline 60 & 10 & 11 & 10 & 11 & 11 & 11 & 10 & 10 & 11 & 11 \\
\hline 40 & 9 & 9 & 9 & 9 & 10 & 10 & 9 & 9 & 9 & 10 \\
\hline 20 & 7 & 7 & 7 & 8 & 8 & 8 & 8 & 7 & 7 & 7 \\
\hline $\mathbf{M}$ & 10 & 10 & 10 & 10 & 10 & 10 & 10 & 10 & 10 & 10 \\
\hline DS & 3 & 3 & 3 & 3 & 3 & 3 & 3 & 3 & 3 & 3 \\
\hline Var & 7 & 8 & 8 & 8 & 8 & 9 & 6 & 8 & 9 & 9 \\
\hline
\end{tabular}

Nota. Elaboración propia

La Tabla 5 contiene los percentiles - PC (20, $40,60,80$ y 100) y los puntajes directos (PD) de cada dimensión del test. El percentil 20 (Bajo) contiene puntajes directos entre 7 y 8 , en el percentil 40 (Regular) los puntajes directos oscilan entre 9 y 10, el percentil 60 (Promedio) tiene puntajes directos entre 10 y 11, el percentil 80 (Bueno) tiene puntajes directos entre 12 y 13, y el percentil 100 (Excelente) tiene 15 como puntaje directo.

\section{DISCUSIÓN}

Pereda (2016) en España construyó un cuestionario que consta de 27 preguntas e identifica 22 habilidades directivas agrupadas en: habilidades instrumentales, habilidades personales $\mathrm{y}$ habilidades transversales. Al respecto, no es pertinente que 22 habilidades sea evaluada con 22 ítems. El Test: Potencial de Habilidades Directivas tiene 50 ítems para evaluar 10 habilidades directivas.

Dincer y Akgum (2015) mediante un estudio fue descriptivo desarrollaron una herramienta de medición para determinar las habilidades directivas en el ámbito educativo de Turquía. Esta herramienta es aplicable solo en el ámbito educativo.

Abadi (2013), en la India, construyó una escala sobre los componentes de las habilidades de gestión integral de la calidad y el proceso de liderazgo. Participaron 250 gerentes mediante un muestreo aleatorio. El coeficiente de consistencia interna de mejora continua fue
0,8 y liderazgo 0,92 . Se hizo uso de estadística descriptiva y el coeficiente de correlación de Pearson.

En el Perú se realizaron investigaciones sobre habilidades directivas para evaluar a jefes y gerentes, todos ellos con muestras menores de 100 encuestados; por lo tanto, el instrumento utilizado es válido para el grupo materia de evaluación, más no puede ser utilizado en otros grupos de Ia población. Ninguno de ellos evalúa el potencial.

En cuanto a evaluar el potencial de habilidades directivas en el Perú, la presente investigación es inédita; por lo tanto, es un aporte instrumental y metodológico a la gestión del talento humano, considerando, además, que se tiene como muestra investigada a 1874 trabajadores. Como toda investigación, esta debe ser contrastada con muestras de mayor tamaño y complementada con investigaciones cualitativas.

\section{CONCLUSIONES}

- Se cumplió satisfactoriamente la construcción del Test: Potencial de Habilidades Directivas.

- La validez mediante opinión de expertos y la aplicación del V de Aiken, el Test tiene una validez de contenido $=$ 0.943, es decir es altamente válido.

- El análisis de la correlación Ítem - Correlación total de elementos corregida 
Diseño y construcción de un instrumento para evaluar el potencial de habilidades directivas de los trabajadores

de los 50 ítems se obtuvo valores entre 0.40 y 0.69 ; por lo tanto, el test tiene validez interna.

- La confiabilidad del Test: Potencial de Habilidades Directivas, según Alfa de Cronbach es 0.977 , lo que significa que tiene una muy fuerte confiabilidad.

- El test cuenta con un baremo.

\section{REFERENCIAS BIBLIOGRÁFICAS}

Abadi, Z. B. G. (2013). Relationship between management skills with the components of total quality management with an emphasis on process improvement and leadership. Kuwait Chapter of the Arabian Journal of Business and Management Review, 2 (11), 6-14. Retrieved from https:// search.proquest.com/docview/1459326322? accountid $=12268$

Alarcón, R. (2013). Métodos y diseños de investigación del comportamiento. Editorial Universitaria.

Anastasi, A. y Urbina, S. (1998). Tests psicológico. Prentice Hall.

Arana, W. (2018). Factores que motivan a los trabajadores en empresas de Lima Metropolitana. Gestión En El Tercer Milenio, 21(41), 75-82. Recuperado a partir de https://revistasinvestiqacion.unmsm.edu.pe/index.php/administrativas/ article/view/15425

Argumedo, D., Nóblega, M. Bárrig, P. y Otiniano, F. (2016). Criterios homologados de investigación en psicología investigaciones instrumentales. Pontificia Universidad Católica del Perú.

Blanchard, K. (2016). Liderazgo al más alto nivel. Editorial Norma.

Budjac, B. (2011). Técnicas de negociación y resolución de conflictos. Pearson.

Cohen, L. y Marrion, L. (2002). Métodos de investigación cuantitativa. La Muralla.

Cohen, A., Stotland, E. y Wolfe, D. (1955). An experimental investigation of need for cognition. Journal of abnormal and social psychology. $\mathrm{N}^{\circ}$ 51, p. 291 - 2914.

Conto Escobar, K. (2020). Construcción y validación de una escala de actitudes hacia palomas domésticas en estudiantes universitarios. Revista de Investigación En Psicología, 22 (2), 287-302. https://doi.org/10.15381/rinvp.v22i2.17427

Dincer, Q. y Akgun, E. (2015). Developing a classroom management skills inventory for preschool teachers, and the correlation of preschool teachers' classroom management skills with different variables. Egitim Ve Bilim, 40(177) Retrieved from https://search.proquest.com/docview/1656565503?accountid=12268

Escurra, L. (1988). Cuantificación de la validez de contenido por criterio de jueces. Revista De Psicología, 6(1-2), 103-111. Recuperado a partir de http://revistas.pucp.edu.pe/index.php/psicologia/article/view/4555

Goleman, D. (2016). Cómo ser un líder. Grupo Z.

González, R. (2005). Creando valor con la gente. Editorial Norma.

Gordon, J. (1997): Comportamiento organizacional. Editorial Prentice-Hall Hispanoamericana.

Hernández, R., Fernández, C. y Baptista, M. (2014). Metodología de Investigación científica. Prentice-Hall Hispanoamericana.

Hernández, R. y Mendoza, C. (2018). Metodología de la Investigación. Prentice-Hall Education.

Infantes, J. (2017). Habilidades directivas y motivación laboral de docentes de secundaria en instituciones educativas privadas - Rímac 2016 (tesis de maestría). Universidad Cesar Vallejo, Lima.

Johnson, C. y Keddy, J. (2015). Manejo de conflicto en el trabajo. 3R Editores.

Lax, D. y Sebenius, J. (2007). Negociación tridimensional. Bogotá: Editorial Norma.

Maxell, J. (2018). El poder de su potencial. New York: Nashville.

Muñiz y Fonseca-Pedrero (2019). Psicothema 2019, 31(1), 7-16. doi:10.7334/psicothema2018.291

Pereda Pérez, F. J., Lopez-Guzman Guzmán, T., Gonzáles Santa Cruz, F. (2014). Las habilidades directivas como ventaja competitiva. El caso del sector público de Ia provincia de Córdoba (España). Intangible Capital, 10(3), 528-561. Recuperado de: https://www.redalyc.org/artículo. oa?id=54932488003

Pereda, F. (2016). Análisis de las habilidades directivas. Estudio aplicado al sector público de la provincia de Córdoba (Tesis doctoral). Universidad de Córdoba, España.

Shein, E. (1982). Psicología de la organización. México: Prentice-hall Hispanoamericana, S.A.

Selye, H. (1956). Stress of Life. San Diego CA: Bennett Books Ltd.

Stoner, J., Freeman, R. y Gilbert, D. (1999). Administración. México: Prentice Hall.

Whetten, D. y Cameron, K. (2016). Desarrollo de habilidades directivas. México: Pearson. 
